\title{
Berufsbedingtes Kontaktekzem nach Anwendung einer Dexpanthenol-haltigen Salbe und Überblick über die IVDK-Daten zu Dexpanthenol
}

\author{
C. Skudlik ${ }^{1}$ \\ A. Schnuch ${ }^{2}$ \\ W. Uter ${ }^{2}$ \\ H. J. Schwanitz ${ }^{1}$
}

\author{
Occupational Contact Dermatitis Caused by Barrier Cream Containing \\ Dexpanthenol and Overview of the Current Dexpanthenol-Data from the \\ IVDK-Database
}

\section{Zusammenfassung}

Eine Kontaktallergie gegenüber Dexpanthenol ist insgesamt relativ selten, wenngleich entsprechende Sensibilisierungen bei bestimmten Subgruppen, wie z. B. Patienten mit Unterschenkelekzemen, gehäuft beobachtet werden. Zwischen 1992 und 1999 wiesen nur 163 von insgesamt 13216 in den dem Informationsverbund Dermatologischer Kliniken (IVDK) angeschlossenen Kliniken mit der Substanz Dexpanthenol getesteter Patienten eine positive Reaktion auf. Die Daten des IVDK zeigen u.a., dass ein gewerbedermatologischer Hintergrund der Testung mit Dexpanthenol eher selten ist, insbesondere fand sich bislang in keinem Fall ein Hinweis auf eine beruflich relevante Dexpanthenol-Sensibilisierung. Erstmals wird nun ein eindeutiges berufsbedingtes allergisches Kontaktekzem gegenüber Dexpanthenol nach Anwendung eines entsprechenden Hautschutzpräparates beschrieben.

\section{Abstract}

Contact allergy to Dexpanthenol is rare, nevertheless it can be observed more frequently in some patient subgroups (e.g. subjects with eczema of the lower leg). Between 1992 and 1999, only 163 out of 13216 patients tested within the Information Network of Departments of Dermatology (IVDK), a multicentre project of Departments of Dermatology, had a positive reaction to Dexpanthenol. Data collected by the IVDK demonstrate that an occupational background of Dexpanthenol contact allergy is apparently very uncommon, actually, no case of Dexpanthenol sensitisation caused by occupational exposure has been identified so far. In this paper we report the first case of allergic contact dermatitis to Dexpanthenol attributable to workplace exposure to a Dexpanthenol containing barrier cream in a junior nurse.

\section{Einleitung}

Dexpanthenol (Bepanthen ${ }^{\circledR}$, Panthenol ${ }^{\circledR}$, Pantothenylalkohol, CAS 81-13-0) ist eine Vorstufe der zur Gruppe der wasserlöslichen Vitamine zählenden Pantothensäure. Es findet eine relativ weite Verbreitung in Externa und Kosmetika, darüber hinaus auch z. B. in systemisch zu verabreichenden Vitamin-Präparaten. Kontaktallergien gegenüber Dexpanthenol wurden überwiegend nach Anwendung entsprechender Externa zur Behandlung von Hauterscheinungen im Rahmen der chronisch-venösen Insuffi- zienz, aber auch z.B. nach Gebrauch Dexpanthenol-haltiger Nasen- und Augensalben bzw. Sonnenschutzmittel beschrieben. Im vorliegenden Beitrag werden der berufsdermatologische Fall einer Kontaktallergie auf eine als Hautschutz verwendete Dexpanthenol-haltige Salbe vorgestellt und die aktuellen Daten des Informationsverbundes Dermatologischer Kliniken (IVDK) zu Dexpanthenol präsentiert.

${ }^{1}$ Dermatologie, Umweltmedizin und Gesundheitstheorie, Universität Osnabrück

${ }^{2}$ Informationsverbund Dermatologischer Kliniken (IVDK) an der Universitäts-Hautklinik Göttingen 
Bei einer 19-jährigen Krankenpflegeschülerin traten ca. 6 Wochen nach Beginn ihrer Ausbildung innerhalb weniger Tage progrediente Rötungen und Juckreiz an beiden Hand- und Fingerrücken auf. Eigenanamnestisch wurde als Auslöser die vermehrte Feuchtarbeit sowie die Einwirkung der verwendeten Pflegelotion bzw. einer kurz zuvor erstmals als Hautschutz angewandten Salbe, welche speziell für das Friseurhandwerk konzipiert wurde (Reamin Spezial ${ }^{\circledR}$ Hand-und Hautsalbe), vermutet. Die Patientin war wegen klinisch erkennbar erhöhter Hautempfindlichkeit zu Beginn ihrer Ausbildung ärztlicherseits auf die Notwendigkeit adäquater Hautschutzmaßnahmen aufmerksam gemacht worden. Die Patientin verwendete allerdings nicht die auf der Station zur Verfügung gestellte Hautschutzcreme, sondern eine speziell für das Friseurgewerbe konzipierte „Hautschutzpflege“-Salbe, welche von einer Bekannten, die als Friseurauszubildende tätig ist, gut vertragen werde.

Klinisch zeigte sich zum Zeitpunkt der erstmaligen Vorstellung an beiden Handrücken mit Streuung auf die Unterarme sowie an sämtlichen Fingern streckseitig ein flächenhaftes, unscharf begrenztes erythematöses Infiltrat. An atopietypischen Kriterien und Stigmata fanden sich klinisch eine periorbitale Verschattung, Perléche, palmare Hyperlinearität und mäßige generalisierte Xerose des Hautorgans. Anamnestisch wurden Rhinokonjunktivitis allergica saisonalis, Metallunverträglichkeit und Ohrrhagaden angegeben. Nach Meidung sämtlicher vermuteter Auslöser und unter einer antiekzematösen Lokaltherapie war das Handekzem innerhalb weniger Tage abgeheilt.

In der Epikutan-Testung mit den Allergenen der Standardreihe, Externa/Grundlagenreihe, Gummireihe und Desinfektionsmittelreihe der Deutschen Kontaktallergie-Gruppe (DKG) sowie der Pflegelotion und der als Hautschutz verwendeten Salbe zeigten sich positive Reaktionen gegenüber Nickel-II-sulfat, Imidazolidinylharnstoff, Phenylquecksilberacetat und der Salbe (je „,+“ nach 48 und 72 Stunden).

\section{Eine Prick-Testung mit Latex war negativ.}

Die Aufschlüsselungstestung und Identifizierung des relevanten Allergens in der als Hautschutz verwendeten Salbe erfolgten unter Nutzung des IDOK-Meldesystems (Informations- und Dokumentationsstelle für Kontaktallergien des Informationsverbundes dermatologischer Kliniken [IVDK], Göttingen). Die IDOK berät hierbei den jeweiligen Kosmetika-Hersteller, nachdem an diesen der Wunsch nach einer Aufschlüsselung wegen einer derartigen positiven Reaktion auf ein Produkt herangetragen wurde, im Hinblick auf die allergologisch relevanten Inhaltsstoffe, die diesbezüglich empfohlenen Testkonzentrationen und das Vehikel. Dadurch ist es möglich, sämtliche Inhaltsstoffe in testfähiger Konzentration zu erhalten und epikutan zu testen [1].

In der Aufschlüsselung der in der Salbe enthaltenen Einzelsubstanzen konnte als Allergen „Panthenol“ (5\% in Vaseline), mit einer jeweils „+“-Reaktion nach 48 und 72 Stunden, identifiziert werden.
Somit bestand eine klinische Relevanz für Dexpanthenol, das in $2 \%$ iger Konzentration in der als „Hautschutzpflege“ verwendeten Salbe enthalten war (und ebenso für Nickel im Sinne einer Modeschmuckunverträglichkeit). Aufgrund dieser Befunde wurde zum einen ein allergisches Kontaktekzem der Hände bei TypIV-Sensibilisierung gegenüber Dexpanthenol und zum anderen ein Z. n. allergischem Kontaktekzem bei Typ-IV-Sensibilisierung gegenüber Nickel diagnostiziert.

Eine klinische Relevanz ließ sich für die Allergene Imidazolidinylharnstoff und Phenylquecksilberacetat hingegen nicht erkennen.

Nach Meidung der Dexpanthenol-haltigen Salbe ist das Handekzem nicht mehr aufgetreten.

\section{IVDK-Daten}

Zwischen Anfang 1992 und Ende 1999 sind in den seinerzeit insgesamt 33 dem Informationsverbund Dermatologischer Kliniken (IVDK) angeschlossenen Kliniken ${ }^{1} 13216$ Patienten mit Dexpanthenol $5 \%$ in Vaseline in der „DKG-Arzneistoff-Reihe“ epikutan getestet worden. Die Ergebnisse sind in Tab. 1 dargestellt; insgesamt zeigten sich 163 - weit überwiegend schwach - positive Testreaktionen (1,2\%).

Tab. 1 Reaktionen im Epikutan-Test 1992-1999 auf Dexpanthenol (IVDK)

\begin{tabular}{|rrr|}
\hline & Anzahl & Prozent \\
\hline gesamt & 13216 & 100,0 \\
\hline neg. & 12874 & 97,4 \\
\hline ?x & 114 & 0,9 \\
\hline ?a & 32 & 0,2 \\
\hline$f$ & 10 & 0,1 \\
+ & 136 & 1,0 \\
\hline++ & 22 & 0,2 \\
\hline+++ & 5 & 0,0 \\
\hline irr. & 23 & 0,2 \\
\hline
\end{tabular}

${ }^{1}$ IVDK-Zentren, die zu dieser Analyse beitrugen (in alphabetischer Reihenfolge): Aachen (H. Dickel), Augsburg (O. Hirschsteiner, A. Ludwig), Berlin B. Franklin (B. Tebbe, R. Treudler), Berlin Charité (B. Laubstein, J. Grabbe, T. Zuberbier), Berlin UKRV (J. Grabbe, T. Zuberbier), Bochum (C. Szliska), Dortmund (P. J. Frosch, B. Pilz, C. Pirker), Dresden (G. Richter), Duisburg (J. Schaller), Erlangen (K. P. Peters, M. Fartasch), Essen (H.-M. Ockenfels, U. Hillen), Göttingen (Th. Fuchs, J. Geier), Graz (W. Aberer, B. Kränke), Halle (G. Gaber, D. Lübbe), Hamburg (M. Kiehn, D. Vieluf), Heidelberg (A. Schulze-Dirks, M. Hartmann), Homburg/Saar (P. Koch), Jena (M. Gebhardt, A. Bauer), Kiel (J. Brasch), Lübeck (J. Kreusch, J. Grabbe), Magdeburg (U. Jappe, E. Weisshaar), Mainz (D. Becker), Mannheim (C. Bayerl), Marburg (I. Effendy), München LMU (B. Przybilla, F. Rueff), München Schwabing (M. Agathos), München TU (J. Rakoski), Nürnberg (I. Müller), Osnabrück (W. Uter), Ulm (H. Gall †), Wuppertal (O. Mainusch, J. Raguz) 
Tab. 2 Demografische Daten des gesamten, zwischen 1992 und 1999 getesteten Patientenkollektivs sowie derjenigen, die mit der Arzneistoff-Reihe getestet wurden, und den Dexpanthenol-Positiven

\begin{tabular}{|c|c|c|c|c|}
\hline & & $\begin{array}{l}\text { gesamt } \\
(n=73977) \rightarrow\end{array}$ & $\begin{array}{l}\text { ektiv und zwei SL } \\
\text { „Arznei-Reihe“ } \\
(n=13306) \rightarrow\end{array}$ & $\begin{array}{l}\text { D.-Positive } \\
(n=163)\end{array}$ \\
\hline $\begin{array}{l}\text { männlich } \\
\text { Berufsdermatose }\end{array}$ & M & 35,4 & 35,1 & 26,8 \\
\hline $\begin{array}{l}\text { „occupational“ } \\
\text { atopisches Ekzem }\end{array}$ & 0 & 14,6 & 10,6 & 9,1 \\
\hline Hand-Ekzem & A & 17,8 & 17,3 & 18,3 \\
\hline & H & 28,2 & 22,1 & 14,6 \\
\hline $\begin{array}{l}\text { Unterschenkel-Ekzem } \\
\text { („Leg Dermatitis“) }\end{array}$ & $\mathbf{L}$ & 8,5 & 19,6 & 29,9 \\
\hline Gesichts-Ekzem („Face Dermatitis“) & $\mathbf{F}$ & 14,7 & 15,7 & 25,6 \\
\hline Alter 40 und darüber & A & 58,0 & 67,4 & 74,4 \\
\hline
\end{tabular}

Fasst man die einfach bis dreifach positiven Reaktionen zusammen, so lässt sich ein konsistenter Zeit-Trend, d. h. eine Zu- oder Abnahme der Dexpanthenol-Sensibilisierungen, nicht erkennen ( $p=0,24$, Cochrane-Armitage Trend-Test).

Die Arzneistoffreihe der DKG wurde unterdurchschnittlich häufig bei Patienten mit Verdacht auf Berufsdermatose eingesetzt, dagegen gezielt bei Patienten mit Unterschenkel-Ekzem (Tab. 2). In der Subgruppe von Patienten mit Dexpanthenol-Kontaktsensibilisierung sind nicht nur diese Patienten, sondern auch solche mit Gesichtsekzem besonders vertreten (Tab. 2).

Im Hinblick auf die klinische Relevanz der Dexpanthenol-Sensibilisierungen fanden sich insgesamt bei 8,4\% der Sensibilisierungen eine klinische Relevanz für ein früheres Ekzemgeschehen und mit 46,7\% ein relativ hoher Anteil an aktuell relevanten Sensibilisierungen.

\section{Kommentar}

Pantothensäure (griechisch pantos: überall) gehört zur Gruppe der wasserlöslichen Vitamine, d.h. sie kann von tierischen Organismen selbst nicht synthetisiert werden. Gleichzeitig ist die Substanz in nahezu allen tierischen und pflanzlichen Bestandteilen nachweisbar. In der Zelle liegt Pantothensäure als Bestandteil des Coenzyms A vor und ist hierüber bedeutsam für den Kohlenhydrat-, Fett- und Eiweißstoffwechsel. Als angemessene Zufuhr von Pantothensäure für einen Erwachsenen werden $6 \mathrm{mg}$ täglich geschätzt, mögliche Quellen für den Menschen sind Fleisch, Fisch, Milch und Milchprodukte, Vollkornerzeugnisse und Hülsenfrüchte $[2,3]$.

Pantothensäure selbst ist thermolabil und lässt sich in saurem oder alkalischem Milieu leicht hydrolysieren. Der Alkohol Panthenol ist diesbezüglich stabiler und wird im Organismus zu Pantothensäure oxidiert. Panthenol ist die Kurzbezeichnung für ( \pm )-2,4-Dihydroxy-N-(3-hydroxypropyl)-3,3-dimethylbutyramid. Die der Pantothensäure entsprechende Vitamin-Aktivität soll hierbei ausschließlich der optisch aktiven D-(+)-Form (Dexpanthenol) zukommen [3,4]. Daher wird diese Substanz ent- sprechend in Externa und Kosmetika, darüber hinaus auch z.B. in systemisch zu verabreichenden Vitamin-Präparaten, eingesetzt.

Bezogen auf das Hautorgan konnte gezeigt werden, dass die Applikation Dexpanthenol-haltiger Externa einen nachhaltigen positiven Effekt auf die epidermale Barrierefunktion ausübt, darüber hinaus konnten positive Effekte zur Wundheilung durch Anstieg der Zahl einwandernder Zellen, Anstieg der Zellteilungsrate und Beeinflussung der Proteinsynthese nachgewiesen werden $[5,6]$.

Allergische Kontaktekzeme wurden beschrieben nach der Anwendung entsprechender Dexpanthenol-haltiger Externa, wie Dermatika zur Therapie von Hauterscheinungen im Rahmen der chronisch-venösen Insuffizienz sowie vereinzelt auch der atopischen Dermatitis, ferner Augen- und Nasen-Salben, antimykotische Externa, Sonnenschutzmittel und Körperpflegemittel inklusive Babypflege-Artikel [3,7-13]. Darüber hinaus wurde die Unterhaltung eines Dexpanthenol-bedingten allergischen Kontaktekzems durch die orale Aufnahme von Pantothensäure beobachtet [8].

Im vorliegenden Fall wurde nunmehr erstmalig auch ein eindeutig berufsbedingtes allergisches Kontaktekzem durch die als Hautschutzpräparat erfolgte Anwendung einer Dexpanthenolhaltigen Salbe beschrieben. Die Auswertung der Daten des IVDK zeigt, dass ein gewerbedermatologischer Hintergrund bei der Testung von Dexpanthenol bzw. allgemein bei den externen Arzneistoffen eher selten vorliegt (Tab. 2), was die Ausnahmerolle der hier kasuistisch beschriebenen beruflichen Sensibilisierung unterstreicht. Hierzu ist ergänzend festzustellen, dass bei den 15 IVDK-Patienten „mit Berufsdermatose“ (Tab. 2) diese Einschätzung nicht auf die Dexpanthenol-Sensibilisierung bezogen war, sondern auch auf anderweitige Sensibilisierungen bzw. das aktuell vorliegende Ekzem insgesamt. In keinem Fall fand sich bei den IVDK-Patienten der Hinweis, dass die DexpanthenolSensibilisierung beruflich relevant war.

Die aktuellen Daten des IVDK weisen aus, dass sich in den letzten Jahren eine wesentliche Änderung der Häufigkeit von Dexpan- 
thenol-Kontaktsensibilisierungen nicht gezeigt hat. Im Verhältnis zu den Allergenen der Standardreihe ist die DexpanthenolAllergie selten. Wenn 1,2\% der im IVDK getesteten Patienten auf die Substanz reagierten, dann beruht diese relativ hohe Zahl auf der selektiven Testung mit der Substanz. Bei der Testung zusammen mit der Standardreihe fanden Hemmer et al. [8] lediglich $0,34 \%$ positive Reaktionen. Andererseits sind bestimmte Subkollektive häufiger betroffen. Hierzu gehören Patienten mit Unterschenkelekzem, die bekanntermaßen besonders leicht gegen Grundlagenbestandteile wie Wirkstoffe von Externa sensibilisierbar sind. Der Befund, dass das Gesichtsekzem deutlich häufiger bei den Dexpanthenol-Positiven vertreten war, unterstützt die Annahme, dass die Exposition nicht nur in Heilsalben, sondern vornehmlich in Kosmetika bestand [14]. Patienten mit atopischem Ekzem waren überraschenderweise nicht häufiger betroffen. Die Deklaration von Dexpanthenol nicht nur in medizinischen Präparaten, sondern auch in Kosmetika (nach INCI: „Panthenol“) erleichtert verglichen mit vielen anderen Allergenen dem Betroffenen die erfolgreiche Allergenmeidung, wie auch in dem hier vorgestellten Fall.

\section{Literatur}

${ }^{1}$ Uter W, Geier J, Lessmann H, Schnuch A. Unverträglichkeitsreaktion gegen Körperpflege- und Haushaltsprodukte: Was ist zu tun. Dtsch Dermatologe 1999; 47: 211 - 214

2 Oberritter H. Prinzipien vollwertiger Ernährung. In: Schauder P, Ollenschläger G (Hrsg). Ernährungsmedizin - Prävention und Therapie. 1 Aufl. München: Urban \& Fischer, 1999: 47-61

${ }^{3}$ Stables GI, Wilkinson SM. Allergic contact dermatitis due to panthenol. Contact Dermatitis 1998; 38: 236-237

${ }^{4}$ Bui-Nguyen MH. Direct determination of D-panthenol in pharmaceutical preparations by ion-pair chromatography. J Chromatogr 1984; 303: $291-295$

${ }^{5}$ Gehring W, Gloor M. Effect of topically applied dexpanthenol on epidermal barrier function and stratum corneum hydration. Results of a human in vivo study. Arzneimittelforschung 2000; 50: 659-663

${ }^{6}$ Weimann BI, Hermann D. Studies on wound healing: effects of calcium D-pantothenate on the migration, proliferation and protein synthesis of human dermal fibroblasts in culture. Int J Vitam Nutr Res 1999; 69: $113-119$

${ }^{7}$ Gollhausen R, Przybilla B, Ring J. Contact allergy to dexpanthenol. Contact Dermatitis 1985; 13: 38

${ }^{8}$ Hemmer W, Bracun R, Wolf-Abdolvahab S, Focke M, Gotz M, Jarisch R. Maintenance of hand exzema by oral pantothenic acid in a patient sensitized to dexpanthenol. Contact Dermatitis 1997; 37: 51

${ }^{9}$ Ippen H. Kontaktallergie auf Dexpanthenol. Dermatosen 1981; 29: 45

${ }^{10}$ Jeanmougin M, Manciet JR, Moulin JP, Blanc F, Pons A, Civatte J. Contact allergy to dexpanthenol in sunscreens. Contact Dermatitis 1988; 18: $240-253$

${ }^{11}$ Keilig W. Kontaktallergie auf Dexpanthenol. Dermatosen 1987; 36: 206-208

${ }^{12}$ Schmid-Grendelmeier P, Wyss M, Elsner P. Contact Allergy to Dexpanthenol - a report of seven cases and review of the literature. Dermatosen 1995; 43: $175-178$

${ }^{13}$ Schulze-Dirks A, Frosch PJ. Kontaktallergie auf Dexpanthenol. Hautarzt 1988; 39: $375-377$

${ }^{14}$ Schnuch A, Geier J, Uter W, Frosch PJ et al. National rates and regional differences in sensitization to allergens of the standard series. Contact Dermatitis 1997; 37: 200-209

\section{Mitgliederversammlung}

Anlässlich der 11. Jahrestagung der Deutschen Dermatologischen Lasergesellschaft LASER 2002 am 25. Mai 2002 wurde im Rahmen der Mitgliederversammlung ein neuer Vorstand der Deutschen Dermatologischen Lasergesellschaft gewählt, der sich wie folgt zusammensetzt:

Dr. med. W. Kimmig, Präsident

Universitäts-Hautklinik Hamburg

Martinistr. 52

20246 Hamburg

Dr. med. M. Schmoll, Vizepräsident

Achternstraße 21

26122 Oldenburg

Dr. med. N. Seeber, 2. Vizepräsident

Wandsbeker Marktstraße 48-50

22041 Hamburg

Kontakt: www.ddl.de, info@ddl.de

\section{Gedächtnisvorlesung}

Prof. Dr. Dr. h.c. Gerd Plewig, Ludwigs-Maximilians-Universität München, hat die 25. Kung-Sun Oh Gedächtnisvorlesung am 27. September 2002 an der Yonsei Universität in Seoul, Korea, gehalten.

Prof. Oh war der Begründer der modernen Dermatologie in Korea und wird jährlich mit dieser Gedächtnisvorlesung geehrt.

\section{Personalia}

Dr. med. Rudolf Rupec und Dr. med. Ralf Wienecke (Dermatologische Klinik der Ludwig-Maximilians-Universität München) haben sich für das Fachgebiet Dermatologie und Venerologie habilitiert und die Lehrbefugnis erhalten. 